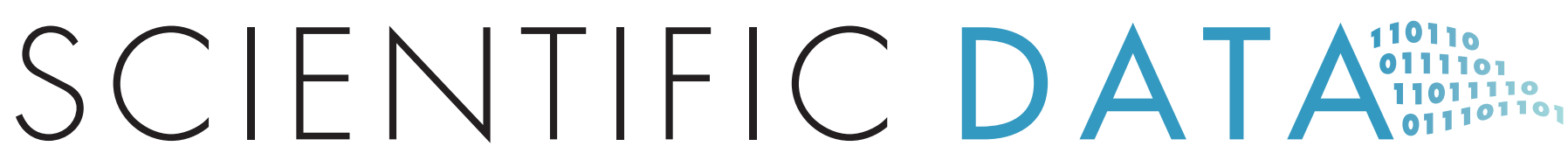

\title{
Corrigendum: Parallel genome-scale loss of function screens in 216 cancer cell lines for the identification of context-specific genetic dependencies
}

Glenn S. Cowley, Barbara A. Weir, Francisca Vazquez, Pablo Tamayo, Justine A. Scott, Scott Rusin, Alexandra East-Seletsky, Levi D. Ali, William F.J. Gerath, Sarah E. Pantel, Patrick H. Lizotte, Guozhi Jiang, Jessica Hsiao, Aviad Tsherniak, Elizabeth Dwinell, Simon Aoyama, Michael Okamoto, William Harrington, Ellen Gelfand, Thomas M. Green, Mark J. Tomko, Shuba Gopal, Terence C. Wong, Hubo Li, Sara Howell, Nicolas Stransky, Ted Liefeld, Dongkeun Jang, Jonathan Bistline, Barbara Hill Meyers, Scott A. Armstrong, Ken C. Anderson, Kimberly Stegmaier, Michael Reich, David Pellman, Jesse S. Boehm, Jill P. Mesirov, Todd R. Golub, David E. Root \& William C. Hahn

Scientific Data 1:140035 doi: 10.1038/sdata.2014.35 (2014); Published 30 September 2014; Updated 11 November 2014

The original version of this Data Descriptor contained a typographical error in the spelling of the author Terence C. Wong, which was incorrectly given as Terrence C. Wong. This has now been corrected in the PDF and HTML versions of the Data Descriptor. 